\title{
Fragile X Mental Retardation Protein: Regulator of Specific mRNAs or Master Regulator of Global Translation?
}

\author{
Denise Cook, Scott A. Cameron, and Emma V. Jones \\ Centre for Research in Neuroscience, Integrated Program in Neuroscience, Department of Neurology and Neurosurgery, The Research Institute of the \\ McGill University Health Centre, Montreal General Hospital, Montreal, Quebec H3G 1A4, Canada \\ Review of Sharma et al.
}

Long-lasting changes in synaptic strength, such as those that occur during late-phase long-term potentiation (LTP) and longterm depression (LTD), require new protein synthesis. This new protein synthesis requires signaling through extracellular signal-regulated kinase (ERK) and mammalian target of rapamycin (mTOR), both of which lead to a global increase in translation that initiates from the $5^{\prime}$ methylguanosine cap found on eukaryotic mRNAs (for review, see Kelleher et al., 2004). Components of the ERK and mTOR signaling pathways, along with mRNAs and translation machinery, are present in dendrites and at synapses, allowing local control of protein synthesis in response to synaptic activity. This local synthesis of proteins is sufficient for synapse-specific changes in synaptic strength (Kang and Schuman, 1996; Huber et al., 2000).

Local protein synthesis is controlled by RNA-binding proteins, including fragile $\mathrm{X}$ mental retardation protein (FMRP). FMRP is an RNA-binding protein that transports, anchors, and represses translation of mRNAs at synapses. Loss of FMRP

Received March 12, 2010; revised April 8, 2010; accepted April 9, 2010.

We thank members of the Centre for Research in Neuroscience Friday Journal Club for their insightful discussions and comments.

Correspondence should be addressed to Denise Cook, Centre for Research in Neuroscience, Montreal General Hospital, 1650 Cedar Avenue L12-409, Montreal, QC H3G 1A4, Canada. E-mail: denise.cook@mail. mcgill.ca.

DOI:10.1523/JNEUROSCI.1298-10.2010

Copyright $\odot 2010$ the authors $\quad 0270-6474 / 10 / 307121-03 \$ 15.00 / 0$ in humans results in fragile X syndrome, characterized by cognitive impairment, sensory deficits, and autism. Mice that lack FMRP show similar impairments and therefore serve as a useful model for research into fragile $\mathrm{X}$ syndrome and the function of FMRP.

Previous studies have shown that loss of FMRP in mice leads to enhanced metabotropic glutamate receptor-dependent long-term depression (mGluR-LTD) at hippocampal CA1 synapses (Huber et al., 2002). Furthermore, mGluR-LTD in mice lacking FMRP occurs in the absence of new protein synthesis, as opposed to mGluR-LTD in wild-type mice, which requires new protein synthesis (Hou et al., 2006; Nosyreva and Huber, 2006). These results have led to the current view that FMRP represses the translation of mRNAs important for mGluR-LTD. This repression is required to bring synaptic activity back to baseline, suggesting that FMRP forms part of a negative feedback loop controlling the extent and duration of mGluR-LTD (for review, see Bear et al., 2004).

The current hypothesis on FMRP function is that it binds to and controls the translation of a subset of brain mRNAs at synapses (Brown et al., 2001). However, in a study recently published in The Journal of Neuroscience, Sharma et al. (2010) found that signaling components of the mTOR pathway, a pathway linked to global increases in translation initiation, were altered in Fmr1 knock-out mice. This suggests that FMRP may act as a regulator of global translation initiation, rather than regulating specific mRNAs.

Using hippocampal or cortical lysates from Fmr1 knock-out mice, Sharma et al. (2010) present four pieces of biochemical evidence supporting an increase in $\mathrm{mTOR}$ activity and one piece of evidence linking FMRP to these increases.

Sharma et al. (2010) found an increase in phosphorylation of mTOR at Ser2448 in hippocampal lysates from both adolescent and adult Fmrl knock-out mice compared to wild-type mice (their Fig. 1). In contrast, mTOR phosphorylation was not significantly increased in cortical lysates. Interestingly, deficits in LTP have been reported in the cortex but not the hippocampus of Fmr1 knock-out mice, suggesting that regional differences in FMRP function may exist (Zhang et al., 2009).

The authors also used three more indirect assays to test for mTOR activity (their Fig. 2). They first quantified the amount of Raptor (a protein that must associate with mTOR for mTOR to exert its downstream effects) that coimmunoprecipitates with mTOR. More Raptor coimmunoprecipitated with mTOR from Fmr1 knock-out lysates than from lysates from wild-type mice. This increase occluded the increase in Raptor-mTOR association induced in wild-type slices by the group I mGluR agonist $(S)$-3,5dihydroxyphenylglycine (DHPG), suggesting that Raptor-mTOR association, 
and thus mTOR activity, is no longer sensitive to mGluR signaling in knock-outs. The authors provided further support for the increase in mTOR activity by demonstrating an increase in the phosphorylation of two key downstream targets of mTOR, S6 kinase and eukaryotic initiation factor $4 \mathrm{E}$ binding protein, in Fmrl knock-out hippocampal slices. Last, the authors demonstrated an increase in the association of key translation initiation factors, eIF4E and eIF4G, in Fmr1 knockout hippocampal slices compared to wild type. Together, these results provide strong support for the hypothesis that mTOR activity is upregulated in Fmrl knock-out mice.

Sharma et al. (2010) next demonstrated a link between the enhanced mTOR activity and protein-synthesis-independent mGluR-LTD observed in Fmrl knock-out mice (their Fig. 3). In agreement with previous reports, they showed that mGluRLTD in wild-type mice required new protein synthesis and was sensitive to rapamycin, a selective inhibitor of $\mathrm{mTOR}$ (Hou and Klann, 2004). In contrast, Fmr1 knock-out mice showed enhanced mGluRLTD that could not be blocked by rapamycin or other protein synthesis inhibitors. The authors attributed this phenotype to elevated mTOR signaling and protein synthesis occurring in the absence of FMRP. They attempted to delineate the pathway further by investigating molecules upstream of mTOR signaling. They found that Akt, a kinase downstream of phosphoinositide 3-kinase (PI3K) and an activator of mTOR signaling, was upregulated in Fmrl knock-out mice (their Fig. 4). In addition, they demonstrated that a PI3K inhibitor reduced mTOR phosphorylation at Ser2448 and restored DHPG-induced mTOR signaling in Fmr1 knock-out slices (their Fig. $5 c$ ). In view of this, it would be interesting to know whether PI3K inhibition also restores the sensitivity of mGluR-LTD to rapamycin in Fmrl knock-out mice.

The results thus far did not address how loss of FMRP causes enhanced mTOR activity. To address this question, Sharma et al. (2010) searched for FMRP target mRNAs that are part of the PI3K-AktmTOR signaling pathway. They found that mRNAs encoding $\mathrm{P} 110 \beta$ and P85, subunits of $\mathrm{PI} 3 \mathrm{~K}$, and $\mathrm{PI} 3 \mathrm{~K}$ enhancer (PIKE-S), were identified as components of an FMRP complex in neurons using microarray technology (Brown et al., 2001). They found that P110 $\beta$ and PIKE-S expression levels were increased in Fmr1 knock-out lysates (their Fig. $5 a$ and Fig.
6). However, they did not examine whether P110 $\beta$ and PIKE-S mRNAs were associated with FMRP or are direct targets of FMRP. To do this, the authors would have to perform RNA electromobility shift assays to demonstrate direct binding of FMRP to these mRNAs.

Together, these results suggest that P110 $\beta$ and PIKE-S mRNAs are not repressed in Fmr1 knock-out mice, leading to increased PI3K/Akt signaling, mTOR activity, and protein synthesis.

These findings leave some interesting outstanding questions regarding FMRP function and the control of mRNA translation. According to the model proposed by Sharma et al. (2010), FMRP represses synthesis of PIKE-S, a nuclear protein and enhancer of nuclear PI3K activity and mTOR signaling. However, how this result fits with studies suggesting that FMRP controls mRNAs at synapses is not discussed. FMRP and Fmr1 mRNA localize to synapses, and FMRP expression in synaptoneurosomes increases in response to mGluR activation (for review, see Antar and Bassell, 2003). This suggests that FMRP plays a role at synapses and that this role may be modified by mGluR signaling. However, it is unclear from the model proposed by Sharma et al. (2010) whether they are suggesting that FMRP represses PIKE-S mRNA at the synapse (their Fig. 7). It is difficult to reconcile the authors' findings with this model because PIKE-S must be synthesized and then transported back to the nucleus before it can enhance PI3K and mTOR activity. Can this nuclear signaling pathway lead to local increases in mTOR activity, as would occur during sparse synaptic activation? And also, is this pathway fast enough to support the rapid increase in protein synthesis that occurs during mGluR-LTD (Huber et al., 2000)? There are therefore many clarifications necessary to make this a feasible model.

There are other pertinent questions that arise from the conclusions of this paper. Until now, it was thought that FMRP controls a subset of mRNAs important for mGluR-LTD (for review, see Bear et al., 2004). How might this be controlled if FMRP controls mTOR, a master regulator of translation? Are there other mRNAbinding proteins, in addition to FMRP, that control this subset of mRNAs? Are the mRNAs required for mGluR-LTD specifically targeted to active synapses to be translated? Or more simply, are all mTOR target mRNAs upregulated, and do active synapses "capture" the LTD proteins? Interestingly, there is a precedent for this type of model, because proteins synthesized during long-lasting LTP can induce long-lasting LTD at other synapses, a mechanism referred to as crosstagging (Kelleher et al., 2004). However, this leads to another question: if mTOR signaling and protein synthesis are required for both long-lasting LTP and LTD, why is there not more convincing evidence for deficits in protein-synthesisdependent LTP in the Fmr1 knock-out hippocampus (Tang et al., 2002; Zhang et al., 2009)? Thus, many unanswered questions about FMRP-mTOR-mGluR-LTD still remain.

The authors' model and these questions might be reconciled if FMRP controls the global translational state of the neuron and not specific mRNAs important for mGluR-LTD. In such a model, in the presence of FMRP, the mTOR pathway would be kept in a low activation state, which means that global translational rates are low. This would be true throughout the neuron. Synaptic activity would act to control the local translational rates through mTOR via a pathway distinct from the FMRP-PIKE-S-nuclear PI3K pathway, possibly through synaptic PIKE-L signaling. In this way, translation could be regulated locally in the dendrite and/or synapses. In the absence of FMRP, the mTOR pathway would be in a high activation state, global translation rates would be high and dysregulated and mGluR-LTD would occur without the need for new protein synthesis. The paper by Sharma et al. (2010) suggests that FMRP may be acting as a master regulator of global translation in neurons.

\section{References}

Antar LN, Bassell GJ (2003) Sunrise at the synapse: the FMRP mRNP shaping the synaptic interface. Neuron 37:555-558.

Bear MF, Huber KM, Warren ST (2004) The mGluR theory of fragile X mental retardation. Trends Neurosci 27:370-377.

Brown V, Jin P, Ceman S, Darnell JC, O’Donnell WT, Tenenbaum SA, Jin X, Feng Y, Wilkinson KD, Keene JD, Darnell RB, Warren ST (2001) Microarray identification of FMRP-associated brain mRNAs and altered mRNA translational profiles in fragile $\mathrm{X}$ syndrome. Cell 107:477-487.

Hou L, Klann E (2004) Activation of the phosphoinositide 3-kinase-Akt-mammalian target of rapamycin signaling pathway is required for metabotropic glutamate receptor-dependent long-term depression. J Neurosci 24:63526361.

Hou L, Antion MD, Hu D, Spencer CM, Paylor R, Klann E (2006) Dynamic translational and proteasomal regulation of fragile $\mathrm{X}$ mental retardation protein controls mGluR- 
dependent long-term depression. Neuron 51: 441-454.

Huber KM, Kayser MS, Bear MF (2000) Role for rapid dendritic protein synthesis in hippocampal mGluR-dependent long-term depression. Science 288:1254-1257.

Huber KM, Gallagher SM, Warren ST, Bear MF (2002) Altered synaptic plasticity in a mouse model of fragile X mental retardation. Proc Natl Acad Sci U S A 99:7746-7750.

Kang H, Schuman EM (1996) A requirement for local protein synthesis in neurotrophin- induced hippocampal synaptic plasticity. Science 273:1402-1406.

Kelleher RJ 3rd, Govindarajan A, Tonegawa S (2004) Translational regulatory mechanisms in persistent forms of synaptic plasticity. Neuron 44:59-73.

Nosyreva ED, Huber KM (2006) Metabotropic receptor-dependent long-term depression persists in the absence of protein synthesis in the mouse model of fragile X syndrome. J Neurophysiol 95:3291-3295.

Sharma A, Hoeffer CA, Takayasu Y, Miyawaki T,
McBride SM, Klann E, Zukin RS (2010) Dysregulation of mTOR signaling in fragile $\mathrm{X}$ syndrome. J Neurosci 30:694-702.

Tang SJ, Reis G, Kang H, Gingras AC, Sonenberg N, Schuman EM (2002) A rapamycin-sensitive signaling pathway contributes to long-term synaptic plasticity in the hippocampus. Proc Natl Acad Sci U S A 99:467-472.

Zhang J, Hou L, Klann E, Nelson DL (2009) Altered hippocampal synaptic plasticity in the FMR1 gene family knockout mouse models. J Neurophysiol 101:2572-2580. 